Neil Louis L. Apale, MD' Joel A. Romualdez, MD' Rodolfo E. Rivera, $\mathrm{MD}^{1}$ Joseph Benjamin M. Lu, MD²

'Department of Otolaryngology Head and Neck Surgery St. Luke's Medical Center

2Department of Radiology St. Luke's Medical Center
Correspondence: Dr. Rodolfo E. Rivera Department of Otolaryngology-Head and Neck Surgery Saint Luke's Medical Center

279 E. Rodriguez Ave., Quezon City 1102 Philippines

Phone: (632) 7230101 local 6246

Email: docodie2003@yahoo.com

Reprints will not be available from the author.

The authors declare that this represents original material that is not being considered for publication elsewhere in full or in part, in print or electronic media; that the manuscript has been read and approved by all the authors, that the requirements for authorship have been met by each authors, and that each author believes that the manuscript represents honest work.

Disclosures: The authors signed disclosures that there are no financial or other (including personal) relationships, intellectual passion, political or religious beliefs, and institutional affiliations that might lead to a conflict of interest.

Presented at Philippine Society of Otolaryngology Head and Neck Surgery, Descriptive Research Contest, September 18 2014, Natrapharm, The Patriot Bldg., KM 18 SLEX, Paranaque City

\section{Pterygopalatine Fossa Infiltration: A Radio-Anatomic Study among Adult Patients in a Tertiary Private Hospital}

\begin{abstract}
Objective: As a guide to the clinical practice of infiltration of local anesthesia into the pterygopalatine fossa via the greater palatine canal, this study sought to determine and record the mean CT scan measurements of the following: 1) palatal mucosal thickness, 2) length and width of greater palatine canal, and 3) length and width of pterygopalatine fossa among adult patients in a private tertiary hospital in Quezon City.
\end{abstract}

\section{Methods:}

Design: Retrospective, Descriptive Study

Setting: Tertiary Private Hospital

Subjects: Paranasal Sinus (PNS) CT Scans of 113 adult patients from January 2014 to May 2014 were reviewed and evaluated. Excluded were images with pathology that distorted the anatomy of the sinuses and surrounding structures.

Results: Our study showed average CT scan measurements of $5.98 \mathrm{~mm}$ palatal mucosal thickness, $16.99 \mathrm{~mm}$ greater palatine canal length, $18.75 \mathrm{~mm}$ pterygopalatine fossa length, $2.37 \mathrm{~mm}$ greater palatine canal width and $2.58 \mathrm{~mm}$ pterygopalatine fossa width. Comparison of average measurements by sex was not statistically significant. There was statistical significance when comparing the right palatal mucosal thickness of $5.86 \mathrm{~mm}$ with the left which was $6.11 \mathrm{~mm}$ with $\mathrm{p}$-value of 0.001 . Comparison between the length of the right pterygopalatine fossa of $18.48 \mathrm{~mm}$ with the left side at $19.01 \mathrm{~mm}$ showed statistical significance with $\mathrm{p}$-value of 0.01 .

Conclusion: As the average measurement of the mucosal palatal thickness combined with the length of the greater palatine canal was $22.97 \mathrm{~mm}$, we recommend bending the needle $23 \mathrm{~mm}$ from the tip in a 45 degree angle for adult patients who will undergo sinus surgery, control of posterior epistaxis, trigeminal nerve block and minor oral cavity surgeries.

Keywords: pterygopalatine fossa infiltration, greater palatine canal, greater palatine foramen, regional anesthesia blocks

The pterygopalatine fossa is a paired cone-shaped depression located posterior to the maxillary sinus. It contains the terminal third of the maxillary artery which gives off the sphenopalatine artery and maxillary nerve.

The greater palatine canal is a passage in the skull which connects the pterygopalatine fossa and the oral cavity. It starts from the inferior end of the pterygopalatine fossa and goes through the sphenoid to reach the palate. It contains the descending palatine artery, vein and palatine nerves.

Philipp J Otolaryngol Head Neck Surg 2015; 30 (1): 24-28

(C) Philippine Society of Otolaryngology - Head and Neck Surgery, Inc 
ORIGINAL ARTICLES

These anatomic structures are clinically important when preparing the nasal cavity preoperatively for endoscopic sinus surgery. Hemostasis can be achieved by infiltrating the pterygopalatine fossa with lidocaineepinephrine solution through the greater palatine canal. This procedure is applicable in septorhinoplasty, management of refractory epistaxis, regional blocks for dental procedures and the treatment of trigeminal neuralgia. ${ }^{1,2,3,4}$

Radio-anatomic measurements of the palatal mucosal thickness, greater palatine canal length and pterygopalatine fossa length are important to determine to accurately identify where to bend the needle used for injection. To the best of our knowledge, there have been no published local reports on CT scan measurements of palatal mucosal thickness, the greater palatine canal and pterygopalatine fossa.

This study aims to provide data of the CT scan measurements of the following: 1) palatal mucosal thickness, 2) length and width of the greater palatine canal, and 3) length and width of the pterygopalatine fossa among adult patients in a private tertiary hospital in the Philippines. Based on the data gathered, we aim to recommend where to bend the needle for instillation of local anesthesia of the pterygopalatine fossa through the greater palatine canal.

\section{METHODS}

A descriptive retrospective study design was utilized in this study. The study was conducted in a private tertiary hospital in Quezon City. A total of 113 computed tomography (CT) scans of the paranasal sinuses were reviewed and evaluated by the author and a radiologist. Each scan was divided and recorded into left and right side totaling 226 cases. We included CT scans of the paranasal sinuses of patients 18-years-old and older done in our institution from January 2014 to May 2014. We excluded scans with pathologies that distorted the anatomy of the sinuses and the surrounding structures such as fractures and masses.

Archived CT images of patients who underwent Functional Endoscopic Sinus Surgery (FESS) Protocol CT scans of the paranasal sinuses were retrieved and reconstructed as specified in the Multi Detector Computed Tomography (MDCT) protocol. Identification, measurement and recording of data were done together with a radiologist.

All PNS FESS protocol CT scans were performed using a 64-slice MDCT scanner (Philips Brilliance iCT, Philips Medical Systems, Ohio, USA).

Axial images, $3 \mathrm{~mm}$ slices in $1.5 \mathrm{~mm}$ intervals were acquired and reconstructed as sagittal and coronal images for each study. Using the Philips Extended Brilliance Workspace software version 4.5.5.51035 (Philips International B.V., Amsterdam, The Netherlands) slice thickness was adjusted with rotation of the angle views reaching 30 degrees in the axial and sagittal planes so that the entire pterygopalatine fossa and greater palatine canal could be viewed in a single slice.

Reconstructed images were reviewed and evaluated to measure the following: 1) thickness of the soft tissue in the palate labelled as $A B$; 2) greater palatine canal length labelled as $B C$ and width; and 3) the pterygopalatine fossa height labelled as CD and width. The greater palatine canal was measured as the segment inferior to the maxillary sinus just prior to where it expanded widthwise becoming the pterygopalatine fossa. The pterygoplatine fossa was defined from the flared up superior extension of the greater palatine canal until it reached the inferior orbital fissure. The overlying oral soft tissue mucosa was measured starting from the free edge of the maxillary prior to becoming the greater palatine canal. The anatomic boundaries of the pterygopalatine fossa were the posterior wall of the maxillary sinus

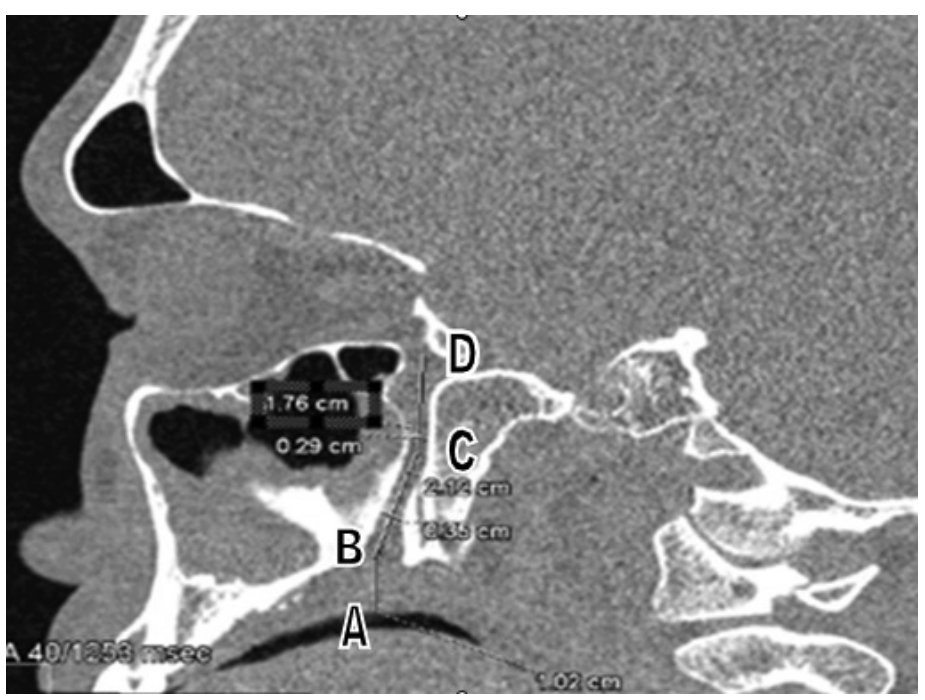

Figure 1. Parasagittal view showing the 1) $A B=$ palatal mucosa, 2) $B C=$ greater palatine canal, and 3 , $C D=$ pterygopalatine fossa.

anteriorly, pterygoid plate posteriorly, inferior orbital wall superiorly and communication with the greater palatine foramen inferiorly. Using the Philips Extended Brilliance Workspace software version 4.5.5.51035 (Philips International B.V., Amsterdam, The Netherlands), measurements were taken by the radiologist with the otolaryngologist as the observer during the process. Figure 1 shows a sample image parasagittal cut with the measurements of palatal mucosa thickness, greater palatine canal and pterygopalatine fossa.

Microsoft Excel Professional 2010 (Microsoft, Redmond, WA, USA) was used for data recording and analysis. The confidence intervals of the average measurements were computed. Independent Sample T-Test was used to analyze the statistical significance of the measurements when compared according to sex and laterality. 


\section{ORIGINAL ARTICLES}

\section{RESULTS}

A total of 113 PNS CT scans taken between January 2014 to May 2014 met the inclusion criteria and were reviewed and evaluated.

The patients' ages ranged from 24 to 77 years old with the mean age of 42.5 years old. Fifty-nine (52.2\%) were females and 54 (47.8\%) were males. Each CT scan study was divided and recorded into left and right side, totalling 226 sides.

Table 1 shows the CT scan measurements of palatal mucosal thickness, length of greater palatine canal and pterygopalatine fossa. The average palatal mucosal thickness was $5.98 \mathrm{~mm}$ with $95 \%$ confidence interval $=5.90-6.06 \mathrm{~mm}$. The average length of the greater palatine canal was $16.99 \mathrm{~mm}$ with $95 \%$ confidence interval $=16.86-17.12 \mathrm{~mm}$. The mean height of pterygopalatine fossa was $18.75 \mathrm{~mm}$ with $95 \%$ confidence interval $=18.54-18.94 \mathrm{~mm}$.

Table 1. CT Scan measurements of palatal mucosa thickness, greater palatine canal length, and pterygopalatine fossa length among adult patients (in millimetres with $95 \%$ confidence interval)

\begin{tabular}{|c|cc|cc|cc|}
\hline & $\begin{array}{c}\text { Palatal Mucosal } \\
\text { Thickness }(\mathbf{m m})\end{array}$ & \multicolumn{2}{|c|}{$\begin{array}{c}\text { Greater Palatine } \\
\text { Canal Length }(\mathbf{m m})\end{array}$} & $\begin{array}{c}\text { Pterygopalatine } \\
\text { Fossa Length }(\mathbf{m m})\end{array}$ \\
\hline Right & 5.86 & $(5.76-5.96)$ & 16.88 & $(16.70-17.06)$ & 18.48 & $(18.22-18.74)$ \\
\hline Left & 6.11 & $(6.00-6.22)$ & 17.11 & $(16.90-17.30)$ & 19.01 & $(18.69-19.31)$ \\
\hline Combined & 5.98 & $(5.90-6.06)$ & 16.99 & $(16.86-17.12)$ & 18.75 & $(18.54-18.94)$ \\
\hline
\end{tabular}

Table 2 shows the CT scan measurements of the width of the greater palatine canal and pterygopalatine fossa. The average width of the greater palatine canal was $2.37 \mathrm{~mm}$ with $95 \%$ confidence interval = 2.30-2.44 mm. The mean width of pterygopalatine fossa is $2.58 \mathrm{~mm}$ with $95 \%$ confidence interval $=2.50-2.66 \mathrm{~mm}$.

Table 2. CT Scan measurement of the widths of the greater palatine canal and pterygopalatine fossa among adult patients (in millimetres with $95 \%$ confidence interval)

\begin{tabular}{|c|c|cc|}
\hline & $\begin{array}{c}\text { Greater Palatine } \\
\text { Canal Width }(\mathbf{m m})\end{array}$ & \multicolumn{2}{|c|}{$\begin{array}{c}\text { Pterygopalatine } \\
\text { Fossa Width }(\mathbf{m m})\end{array}$} \\
\hline Right & $2.42(2.32-2.52)$ & 2.62 & $(2.51-2.73)$ \\
\hline Left & $2.32(2.21-2.43)$ & 2.53 & $(2.42-2.64)$ \\
\hline Combined & $2.37(2.30-2.44)$ & 2.58 & $(2.50-2.66)$ \\
\hline
\end{tabular}

Table 3. CT Scan measurement of palatal mucosa thickness, greater palatine canal length, and pterygopalatine fossa length among adult male patients (in millimetres with 95\% confidence interval)

\begin{tabular}{|c|cc|cc|c|c|}
\hline & \multicolumn{2}{|c|}{ Palatal Mucosal } & \multicolumn{2}{|c|}{$\begin{array}{c}\text { Greater Palatine } \\
\text { Thickness }(\mathbf{m m})\end{array}$} & $\begin{array}{c}\text { Pterygopalatine } \\
\text { Canal Length }(\mathbf{m m})\end{array}$ & Fossa Length $(\mathbf{m m})$ \\
\hline Right & 5.97 & $(5.82-6.12)$ & 16.92 & $(16.67-17.17)$ & 18.63 & $(18.21-19.05)$ \\
\hline Left & 6.14 & $(5.99-6.29)$ & 17.11 & $(16.82-17.40)$ & 19.14 & $(18.66-19.62)$ \\
\hline Combined & 6.05 & $(5.94-6.16)$ & 17.02 & $(16.83-17.21)$ & 18.89 & $(18.57-19.21)$ \\
\hline
\end{tabular}

Table 3 shows the CT scan measurements of palatal mucosal thickness, length of greater palatine canal and pterygopalatine fossa among males. The average palatal mucosal thickness was $6.05 \mathrm{~mm}$ with $95 \%$ confidence interval $=5.94-6.16 \mathrm{~mm}$. The average length of the greater palatine canal was $17.02 \mathrm{~mm}$ with $95 \%$ confidence interval = $16.83-17.21 \mathrm{~mm}$. The mean height of pterygopalatine fossa is $18.89 \mathrm{~mm}$ with 95\% confidence interval $=18.57-19.21 \mathrm{~mm}$.

Table 4 shows the CT scan measurements of the width of the greater palatine canal and pterygopalatine fossa among males. The average width of the greater palatine canal was $2.43 \mathrm{~mm}$ with $95 \%$ confidence interval $=2.31-2.55 \mathrm{~mm}$. The mean width of pterygopalatine fossa was $2.66 \mathrm{~mm}$ with $95 \%$ confidence interval $=2.55-2.77 \mathrm{~mm}$.

Table 4. CT Scan measurements of greater palatine canal width and pterygopalatine fossa width among adult male patients (in millimetres with 95\% confidence interval)

\begin{tabular}{|c|cc|cc|}
\hline & \multicolumn{2}{|c|}{$\begin{array}{c}\text { Greater Palatine } \\
\text { Canal Width (mm) }\end{array}$} & \multicolumn{3}{c|}{$\begin{array}{c}\text { Pterygopalatine } \\
\text { Fossa Width (mm) }\end{array}$} \\
\hline Right & 2.53 & $(2.39-2.67)$ & 2.78 & $(2.64-2.92)$ \\
\hline Left & 2.33 & $(2.14-2.52)$ & 2.54 & $(2.37-2.69)$ \\
\hline Combined & 2.43 & $(2.31-2.55)$ & 2.66 & $(2.55-2.77)$ \\
\hline
\end{tabular}

Table 5 shows the CT scan measurements of the palatal mucosal thickness, length of the greater palatine canal and pterygopalatine fossa among females. The average palatal mucosal thickness was $5.93 \mathrm{~mm}$ with $95 \%$ confidence interval $=5.83-6.03 \mathrm{~mm}$. The average length of the greater palatine canal was $16.97 \mathrm{~mm}$ with $95 \%$ confidence interval $=16.78-17.16 \mathrm{~mm}$. The mean height of the pterygopalatine fossa was $18.61 \mathrm{~mm}$ with $95 \%$ confidence interval $=18.63-18.88 \mathrm{~mm}$.

Table 5. CT Scan measurements of palatal mucosa thickness, greater palatine canal length, and pterygopalatine fossa length among adult female patients (in millimetres with $95 \%$ confidence interval)

\begin{tabular}{|c|cc|cc|cc|}
\hline & \multicolumn{2}{|c|}{$\begin{array}{c}\text { Palatal Mucosal } \\
\text { Thickness }(\mathbf{m m})\end{array}$} & \multicolumn{2}{|c|}{$\begin{array}{c}\text { Greater Palatine } \\
\text { Canal Length }(\mathbf{m m})\end{array}$} & \multicolumn{2}{c|}{$\begin{array}{c}\text { Pterygopalatine } \\
\text { Fossa Length }(\mathbf{m m})\end{array}$} \\
\hline Right & 5.77 & $(5.63-5.91)$ & 16.84 & $(16.59-17.09)$ & 18.34 & $(18.02-18.66)$ \\
\hline Left & 6.10 & $(5.94-6.24)$ & 17.10 & $(16.82-17.38)$ & 18.88 & $(18.49-19.27)$ \\
\hline Combined & 5.93 & $(5.83-6.03)$ & 16.97 & $(16.78-17.16)$ & 18.61 & $(18.36-18.88)$ \\
\hline
\end{tabular}

Table 6 shows the CT scan measurements of width of the greater palatine canal and pterygopalatine fossa among females. The average width of the greater palatine canal was $2.31 \mathrm{~mm}$ with $95 \%$ confidence interval $=2.22-2.4 \mathrm{~mm}$. The mean width of the pterygopalatine fossa was $2.5 \mathrm{~mm}$ with $95 \%$ confidence interval $=2.38-2.62 \mathrm{~mm}$.

The average width of the greater palatine canal was $2.37 \mathrm{~mm}(95 \% \mathrm{Cl}$ $=2.3-2.44$ ) and the average width of the pterygopalatine fossa was 2.58 $\mathrm{mm}(95 \% \mathrm{Cl}=2.5-2.66)$. The average palatal mucosal thickness among 
ORIGINAL ARTICLES

Table 6. CT Scan measurements of greater palatine canal width and pterygopalatine fossa width among adult female patients (in millimetres with 95\% confidence interval)

\begin{tabular}{|c|cc|cc|}
\hline & \multicolumn{2}{|c|}{$\begin{array}{c}\text { Greater Palatine } \\
\text { Canal Width }(\mathbf{m m})\end{array}$} & \multicolumn{2}{|c|}{$\begin{array}{c}\text { Pterygopalatine } \\
\text { Fossa Width (mm) }\end{array}$} \\
\hline Right & 2.32 & $(2.19-2.45)$ & 2.47 & $(2.30-2.64)$ \\
\hline Left & 2.31 & $(2.17-2.45)$ & 2.53 & $(2.37-2.69)$ \\
\hline Combined & 2.31 & $(2.22-2.40)$ & $2.50 \quad(2.38-2.62)$ \\
\hline
\end{tabular}

males was $6.05 \mathrm{~mm}(95 \% \mathrm{Cl}=5.94-6.16)$ which was thicker compared to females at $5.93 \mathrm{~mm}(95 \% \mathrm{Cl}=5.83-6.03)$ with $\mathrm{p}$-value of 0.11 . There was no statistically significant difference.

The average length of the greater palatine canal among males was $17.02 \mathrm{~mm}(95 \% \mathrm{Cl}=16.83-17.21)$ which was longer compared to females with $16.97 \mathrm{~mm}(95 \% \mathrm{Cl}=16.78-17.16)$ with $\mathrm{p}$-value 0.73 . There was no statistically significant difference.

The average length of the pterygopalatine fossa among males was $18.89 \mathrm{~mm}(95 \% \mathrm{Cl}=18.57-19.21)$ which was longer compared to females at $18.61 \mathrm{~mm}(95 \% \mathrm{Cl}=18.36-18.88)$ with $\mathrm{p}$-value 0.19 . There was no statistically significant difference.

There was a statistically significant difference in the average measurements based on laterality. The average palatal mucosal thickness of the right side was $5.86 \mathrm{~mm}(95 \% \mathrm{Cl}=5.76-5.96)$ which was thinner than the left with average thickness of $6.11 \mathrm{~mm}(95 \% \mathrm{Cl}=$ 6-6.22) with $p$-value of 0.001 .

The average length of the greater palatine canal of the right side was $16.88 \mathrm{~mm}(95 \% \mathrm{Cl}=16.7-17.06)$ which was shorter compared to the left at $17.11 \mathrm{~mm}(95 \% \mathrm{Cl}=16.9-17.3)$ with $\mathrm{p}$-value of 0.09 . There was no statistically significant difference.

The average length of the pterygopalatine fossa of the right side was $18.48 \mathrm{~mm}(95 \% \mathrm{Cl}=18.22-18.74)$ which was shorter than the left at $19.01 \mathrm{~mm}(95 \% \mathrm{Cl}=18.69-19.31)$ with $\mathrm{p}$-value of 0.01 which was not statistically significant.

The average width of the greater palatine canal of both sides was compared with a $p$-value of 0.1 which was not statistically significant. When compared according to sex, the $p$-value was 0.13 which was not statistically significant either.

The average width of the pterygopalatine fossa was compared based on its laterality with a $p$-value of 0.29 , which was not statistically significant. Comparison according to sex had a p-value of 0.05 which was statistically significant.

\section{DISCUSSION}

Accurate and correct identification of the nasal and paranasal anatomy is of utmost importance in endoscopic sinus surgery to avoid complications. It is very difficult achieve this when there is bleeding in the surgical field. Bleeding is an important problem during endoscopic sinus surgery. Different methods are practiced to attain hemostasis including local application of decongestant-soaked cotton or cottonoid inside the nasal cavity, infiltration of lidocaine:epinephrine solution into the axilla of the middle turbinate and transpalatal infiltration of lidocaine:epinephrine solution into the pterygopalatine fossa through the greater palatine canal. Wormald ${ }^{4}$ concluded that the infiltration of vasoconstricting solution to this fossa through the greater palatine canal demonstrated significant reduction of intraoperative bleeding during FESS. A study on the effect of greater palatine canal injection on estimated blood loss in sinus surgery showed a decrease in estimated blood loss in patients who had a greater palatine canal injection. ${ }^{5}$ Another study suggested that $2 \mathrm{ml}$ of local anesthetic combined with adrenaline should be injected barely to the pterygopalatine fossa to achieve effective hemostasis. ${ }^{6}$

The CT scan measurements of the palatal mucosal thickness, greater palatine canal length and width and pterygopalatine fossa length and width of adult patients may help surgeons prepare local anesthetic solution with the appropriate needle gauge and correct measurement of where to bend the needle from its tip. Bending the needle in the correct area may help prevent the tip of the needle being inserted further and possibly injuring the nerve and artery in the pterygopalatine fossa (which can result in complications such as intravascular injection with associated cardiovascular side effects, blindness due to vasoconstriction of the ophthalmic artery, infraorbital nerve injury, infratemporal fossa abscess and meningitis).

In our study, the average palatal mucosa thickness was $5.98 \mathrm{~mm}$ $(95 \% \mathrm{Cl}=5.9-6.06)$. In the study by Douglas and Wormald ${ }^{6}$ the palatal mucosa thickness average measurement was $6.9 \mathrm{~mm}(95 \% \mathrm{Cl}=6.2$ 7.6). This suggests a difference in average measurements between Caucasians and our sample. In a study by Methathrathip et al., the mean palatal mucosal thickness among 55 Thai cadaver heads was $6.7 \mathrm{~mm}(95 \% \mathrm{Cl}=4.4-9)$, which is comparable with the results of our study.

In our study, the average length of the greater palatine canal measured was $16.99 \mathrm{~mm}(95 \% \mathrm{Cl}=16.86-17.12)$. Douglas and Wormald ${ }^{6}$ reported an average of $18.5 \mathrm{~mm}(95 \% \mathrm{Cl}=17.9-19.1)$ among Caucasian skulls. Methathrathip et al. ${ }^{7}$ reported an average length of greater palatine canal combined with pterygopalatine fossa of $29.7 \mathrm{~mm}(95 \%$ $\mathrm{Cl}=25.5$ - 33.9) among Thai skulls. Howard-Swirzinski et al. ${ }^{8}$ studied Caucasian patients and measured the greater palatine canal length using Cone Beam Computed Tomography with an average of $29 \mathrm{~mm}$ $(95 \% \mathrm{Cl}=26-32)$. Two other studies reported lengths of $14.6 \mathrm{~mm}$ (9-20 $\mathrm{mm}$ ) and $31.8 \mathrm{~mm}$ (30.45-33.19), respectively. ${ }^{9,10}$

The mean length of pterygopalatine fossa in our study was 18.75 
$\mathrm{mm}(95 \% \mathrm{Cl}=18.54-18.94)$. Douglas and Wormald ${ }^{6}$ reported $21.6 \mathrm{~mm}$ (95\% Cl $=20.7-22.5)$.

Table 7 shows the results of our studies compared to other published reports. There was a statistically significant difference with a $p$-value of $<0.0001$. The differences of the means could be due to: 1) difference of the anatomy of Asians compared to Caucasians; and 2) most of the studies published used cadavers as their population while our study used strictly CT scan images and did not use cadavers.

Table 7. Result Comparisons with Other Published Studies

\begin{tabular}{|c|c|c|c|}
\hline & $\begin{array}{l}\text { Palatal Mucosal } \\
\text { Thickness (mm) }\end{array}$ & $\begin{array}{c}\text { Greater Palatine } \\
\text { Canal Length }(\mathrm{mm})\end{array}$ & $\begin{array}{l}\text { Pterygopalatine } \\
\text { Fossa Length }(\mathrm{mm})\end{array}$ \\
\hline Our Study (2014) & $5.98 \quad(5.90-6.06)$ & $16.99(16.86-17.12)$ & $18.75(18.54-18.94)$ \\
\hline $\begin{array}{c}\text { Douglas and } \\
\text { Wormald } 2006\end{array}$ & 6.9 (6.2-7.6) & 18.5 (17.9-19.1) & $21.6(20.7-22.5)$ \\
\hline $\begin{array}{l}\text { Methathrathip et } \\
\text { al. } 2005\end{array}$ & & & $\begin{array}{c}29.7(25.5-33.9) \\
(G P C+P P F)\end{array}$ \\
\hline $\begin{array}{l}\text { Howard- } \\
\text { Swirzinski et al. } \\
2010\end{array}$ & & $29 \quad(26-32)$ & \\
\hline Hassman 1989 & & $14.6(9-20)$ & \\
\hline $\begin{array}{l}\text { Sheikhi et al. } \\
2013\end{array}$ & & $31.8 \quad(30.45-33.19)$ & \\
\hline
\end{tabular}

There have been several published reports recommending where to bend the needle. The recommendation of Douglas and Wormald 6 is to bend the needle $25 \mathrm{~mm}$ from the tip at a 45 degree angle because the greater palatine canal and hard palate form an angle of approximately 60 degrees. This would facilitate the passage of the needle through the canal and to prevent the needle from penetrating too far the pterygopalatine fossa. This was supported by a study in Thailand which reported that the mean angle of greater palatine canal in relationship with the hard palate is $57.9 \pm 5.8$ degrees in Thai skulls. ${ }^{7}$

In our study, we did a radio-anatomic measurement of the width of the greater palatine canal and pterygopalatine fossa. The average width of the greater palatine canal was $2.37 \mathrm{~mm}(95 \% \mathrm{Cl}=2.3-2.44)$ and pterygopalatine fossa was $2.58 \mathrm{~mm}(95 \% \mathrm{Cl}=2.5-2.66)$. There are several published reports regarding the diameter of the greater palatine canal. Most studies were done with cadavers. Nimigean et al. ${ }^{11}$ studied European skulls and measured the antero-posterior diameter of the greater palatine canal as $4.90 \mathrm{~mm}(95 \% \mathrm{Cl}=4-5.8)$. Methathrathip et al.' studied Thai skulls and measured the greater palatine canal at a diameter of $2.70 \mathrm{~mm}(95 \% \mathrm{Cl}=2.65-3.2)$.

The data from this study may be useful for the preoperative infiltration of lidocaine:epinephrine solution into the pterygopalatine fossa via the greater palatine canal-- a technique used to achieve hemostasis during endoscopic sinus surgery and other procedures as well (e.g. control of epistaxis, treatment of trigeminal neuralgia, local anesthesia for oral procedures).

Measuring the palatal mucosal thickness, the length and width of greater palatine canal and the length and width of pterygopalatine fossa in preoperative PNS CT scans is recommended for the otolaryngologist to be able to properly prepare the needle for local injection of lidocaine and epinephrine solution for hemostasis prior to sinus surgery.

With the average measurement of the mucosal palatal thickness combined with the length of the greater palatine canal at $22.97 \mathrm{~mm}$, we recommend bending the needle for local anesthesia $23 \mathrm{~mm}$ from its tip in a 45 degree angle for local patients who will undergo sinus surgery, trigeminal nerve block, control of posterior epistaxis and minor oral cavity and dental procedures under local anesthesia.

The differences of the results of our study compared to other published reports could be attributed to differences in subjects studied (e.g. cadavers or skulls vs CT scan images) and anatomic differences between Asians and Caucasians. Measuring the angle between the palatal mucosa and greater palatine canal was not done in this study and is recommended for future research.

\section{REFERENCES}

1. Nader A, Kendall MC, De Oliveria GS, Chen JQ, Vanderby B, Rosenow JM, et al. Ultrasound-guided trigeminal nerve block via the pterygopalatine fossa: an effective treatment for trigeminal neuralgia and atypical facial pain. Pain Physician. 2013 Sep-Oct: 16(5): 37-45.

2. Broering R, Reader A, Drum M, Nusstein J, Beck M. A prospective, randomized comparison of the anesthetic efficacy of the greater palatine and high tuberosity second division nerve blocks. $J$ Endod. 2009 Oct; 35(10):1337-1342.

3. Bharadwaj VK, Novotny GM. Greater palatine canal injection: an alternative to the posterior nasal packing and arterial ligation in epistaxis. J Otolaryngol. 1986 Apr; 15(2): 94-100.

4. Wormald PJ, Athanasiadis T, Rees G, Robinson S. An evaluation of effect of pterygopalatine fossa injection with local anaesthetic and adrenalin in the control of nasal bleeding during endoscopic sinus surgery. Am J Rhinol. 2005 May-Jun; 19(3): 288-292.

5. Eloy JA, Kovalerchik O, Bublik M, Ruiz JW, Casiano RR. Effect of greater palatine canal injection on estimated blood loss during endoscopic sinus surgery. Am J Otolaryngol. 2014 Jan-Feb; 35(1): 1-4.

6. Douglas $\mathrm{R}$, Wormald PJ. Pterygopalatine fossa infiltration through the greater palatine foramen: where to bend the needle. Laryngoscope. 2006 Jul; 116(7): 1255-1257.

7. Methathrathip D, Apinhasmit W, Chompoopong S, Lertsirithong A, Ariyawatkul T, Sangvichien S. Anatomy of greater palatine foramen and canal and pterygopalatine fossa in Thais: considerations for maxillary nerve block. Surg Radiol Anat. 2005 Dec; 27(6): 511-516.

8. Howard-Swirzinski K, Edwards PC, Saini TS, Norton NS. Length and geometric patterns of the greater palatine canal observed in cone beam computed tomography. Int J Dent. 2010; 2010:292753: 1-6.

9. Hassmann H. Form, Mabe und Verlaufe der Schadelkana le. In: Lang J, ed. Clinical Anatomy of the Nose, Nasal Cavity and Paranasal Sinuses. New York: Thieme Medical Publishers: 1989:114

10. Sheikhi M, Zamaninaser A, Jalalian F. Length and anatomic routes of the greater palatine canal as observed by cone beam computed tomography. Dent Res J. 2013 Mar; 10(2): 155-161.

11. Nimigean V, Nimigean VR, Butincu L, Salavastru DI, Podoleanu L. Anatomical and clinica considerations regarding the greater palatine foramen. Rom J Morphol Embryol. 2013; 54(3 Suppl): 779-783. 